\title{
Formal Extension of the Whitney Functor and Duality
}

\author{
Ana Rita Martins (*) - Teresa Monteiro Fernandes (**) $\left({ }^{1}\right)$
}

ABSTRACT - On a complex manifold we introduce the formal extension of the Whitney functor and the algebraic extension of the tempered cohomology functor, and prove a natural topological duality between them.

\section{Introduction}

In [7], Kashiwara and Schapira introduced the Whitney and the tempered cohomology functors on the subanalytic site $X_{s a}$ associated to a complex manifold $X$, giving a meaning to $\mathcal{C}_{X_{s a}}^{\infty, \mathrm{w}}$ and to $\mathcal{O}_{X_{s a}}^{\mathrm{W}}$ (Whitney $\mathcal{C}^{\infty}$ and holomorphic functions), to $\mathcal{D} b_{X_{s a}}^{t}$ and to $\mathcal{O}_{X_{s a}}^{t}$ (tempered distributions and tempered holomorphic functions) as sheaves (in the derived sense) on $X_{s a}$. We also refer to [9] for a detailed study on sheaves on the subanalytic site.

Let $\mathcal{D}_{X}$ denote the sheaf of linear differential operators on $X$.

The duality theorem of Kashiwara and Schapira (Theorem 6.1 of [7]) states that taking global sections of the Whitney functor gives a complex of topological C-vector spaces of type $\mathrm{FN}$, in duality with the complex of compactly supported sections of tempered cohomology, this last complex

(*) Indirizzo dell'A.: Faculdade de Engenharia da Universidade Católica Portuguesa, Estrada Octávio Pato, 2635-631 Rio-de-Mouro Portugal.

E-mail: ritamartins@fe.lisboa.ucp.pt

(**) Indirizzo dell'A.: Centro de Matemática e Aplicações Fundamentais e Departamento de Matemática da FCUL, Complexo 2, 2 Avenida Prof. Gama Pinto, 1649-003, Lisboa Portugal.

E-mail: tmf@ptmat.fc.ul.pt

( $\left.{ }^{1}\right)$ The research of the author was supported by Fundação para a Ciência e Tecnologia and Programa Ciência, Tecnologia e Inovação do Quadro Comunitário de Apoio.

Mathematics Subject Classification. Primary: 32C28, 46A20; Secondary: 18E30, 46A13. 
being of topological DFN type. Thus they generalized to the framework of $\mathcal{D}_{X}$-modules the classical duality between $\mathcal{C}^{\infty}$-functions and distributions with compact support.

Influenced by several papers on Deformation Quantization, it became a natural question to extend various results in $\mathcal{D}$-module theory to the case of the formal extension of $\mathcal{D}_{X}$ by a parameter $\hbar$, that is, to $\mathcal{D}_{X}[[\hbar]]$-modules. We refer, in particular, [1] and [10].

In this paper, we are interested in extending the above described topological duality to this new framework. The topological space $\mathrm{C}[[\hbar]]:=\mathbb{C}^{\hbar}$ is a $\mathrm{FN}$ space. Its topological dual is classically known as the space $\mathrm{C}[\hbar]$, but here, as we shall show, it is more natural to consider its dual as being the quotient of the fraction field $\mathrm{C}((\hbar))$ by $\mathrm{C}[[\hbar]]$, $\mathbb{C}((\hbar)) / \mathbb{C}[[\hbar]]$, which will be denoted $\mathbb{C}^{[\hbar]}$ for short. Clearly $\mathbb{C}^{[\hbar]}$ is isomorphic (as a $\mathrm{C}[[\hbar]]$-module with torsion) to the polynomial ring $\mathrm{C}\left[\hbar^{-1}\right]$ with the relations $\hbar \times 1=0, \hbar \times \hbar^{-1}=0, \hbar \times \hbar^{-j}=\hbar^{-j+1}$, for $j>1$. For $f \in \mathbb{C}^{\hbar}$ and $g \in \mathbb{C}^{[\hbar]}$ the duality is then given by $\langle f, g\rangle=\operatorname{Res}_{\hbar=0} f g$.

We shall need to work with the (left) derived functor of the tensor product of sheaves of $\mathrm{C}^{\hbar}$-modules by the $\mathrm{C}^{\hbar}$-module $\mathrm{C}^{[\hbar]}$. The extension (of a $C[[\hbar]]-m o d u l e)$ by $\mathrm{C}^{[\hbar]}$ will be called "algebraic extension" for short.

After reviewing notations and necessary results on the subanalytic site in Section 2 and on topological duality in Section 3, Section 4 is dedicated to introduce and study the formal extension of the Whitney functor, as a functor on the category of $\mathbb{R}$-constructible objects over $\mathbb{C}^{\hbar}$. Inspired by the formal extension of the functor of tempered cohomology performed by [1], we use the theory of sheaves on the subanalytic site and define the sheaves $\mathcal{C}_{X_{s a}, \mathrm{w}, \hbar}$ (of Whitney $\mathcal{C}^{\infty, \hbar}$ functions) and $\mathcal{O}_{X_{s a}}^{\mathrm{w}, \hbar}$ (of Whitney holomorphic functions) on $X_{s a}$.

Section 5 is dedicated to introduce and study the algebraic extension functor. Namely, we introduce the notion of cohomologically $\hbar$-torsion object, as a kind of dual of the notion of cohomologically $\hbar$-complete introduced in [8]. We construct the algebraic extension of tempered cohomology on the category of $\mathbb{R}$-constructible objects over $\mathbb{C}^{\hbar}$, following the same technique as in the preceding section, and we define the sheaves $D b_{X_{s a}}^{t,[\hbar]}$ and $\mathcal{O}_{X_{s a}}^{t,[\hbar]}$.

We obtain comparison results (cf. Proposition 4.7, Proposition 5.13) for formal and algebraic extension of regular holonomic $\mathcal{D}^{\hbar}$-modules as an application of the results in [7].

In Section 6 we state and prove the topological duality in the framework of the new functors. More precisely, in Proposition 6.1 we prove that taking global sections of the formal extension of the Whitney functor still leads to a complex of FN spaces, and that taking compactly supported sections of 
the algebraic extension of the tempered cohomology still leads to a complex of DFN spaces. Moreover we obtain a topological duality between these complexes.

Theorem 6.2 establishes the topological FN type of the complex of solutions of coherent $\mathcal{D}_{X}^{\hbar}$-modules with values in the formal extension of the Whitney product, as well as the DFN type of the complex of compactly supported solutions of coherent $\mathcal{D}_{X}^{\hbar}$-modules with values in the algebraic extension of the tempered holomorphic functions. By Proposition 6.1 these complexes are mutually dual.

It is a pleasure to thank M. Kashiwara and P. Schapira for their enlightening suggestions. We also thank Luca Prelli for his comments on the subanalytic site and Stephane Guillermou for useful discussions.

\section{Review and complements on sheaves on the subanalytic site and formal extensions}

For the background on sheaves on the subanalytic site and Propositions 2.1 and 2.2 below we refer to [5] (also to [9] for a detailed study). For the background on formal extensions we refer to [8] and also [1] for the formal extension of the temperate cohomology functor.

\section{1 - Sheaves on the subanalytic site}

Let $\mathbb{K}$ be a unital Noetherian ring which we assume to have finite global dimension. In practice, throughout this paper, $\mathbb{K}$ will be $\mathbb{C}$ or $\mathbb{C}[[\hbar]]$.

Given a sheaf $\mathcal{R}$ of $\mathbb{K}$-algebras on a topological space $X$, or more generally, on a site, we denote by $\operatorname{Mod}(\mathcal{R})$ the category of left $\mathcal{R}$-modules. We use the notations $D(\mathcal{R})$ for the derived category of $\operatorname{Mod}(\mathcal{R})$ and $D^{b}(\mathcal{R})$ for its bounded derived category. We denote by $D_{\mathrm{coh}}^{b}(\mathcal{R})$ the full triangulated subcategory of $D^{b}(\mathcal{R})$ consisting of objects with coherent cohomology.

For a real analytic manifold $X$, we denote by $\operatorname{Mod}_{\mathbb{R}-c}\left(\mathbb{K}_{X}\right)$ (resp. $\operatorname{Mod}_{\mathbb{R}-c}^{c}\left(K_{X}\right)$ ) the category of R-constructible sheaves (resp. with compact support) of $\mathbb{K}$-modules. We denote $D_{\mathrm{R}-c}^{b}\left(\mathbb{K}_{X}\right)$ the bounded derived category of $\operatorname{Mod}_{\mathbb{R}-c}\left(\mathbb{K}_{X}\right)$ of objects having R-constructible cohomology. For $F \in D_{\mathbb{R}-c}^{b}\left(\mathbb{K}_{X}\right)$ we note $D^{\prime}(F)$ the object $R \mathcal{H} \operatorname{Hom}_{\mathbb{K}_{X}}\left(F, \mathbb{K}_{X}\right)$.

We also denote by $\mathcal{D} b_{X}$ the sheaf of Schwartz distributions, by $\mathcal{C}_{X}^{\infty}$ the sheaf of $\mathcal{C}^{\infty}$ functions, by $\mathcal{A}_{X}$ the sheaf of real analytic functions and by $\mathcal{A}_{X}^{v}$ the sheaf of real analytic densities. 
Let $X_{s a}$ denote the associated subanalytic site to a real analytic manifold $X$, that is, the presite $\operatorname{Op}\left(X_{s a}\right)$ of subanalytic open subsets of $X$ endowed with the Grothendieck topology for which the coverings are those admitting a finite sub-covering. Recall that one has a natural morphism of sites

$$
\rho: X \rightarrow X_{s a}
$$

which induces functors

$$
\operatorname{Mod}\left(\mathrm{C}_{X}\right) \underset{\rho^{-1}}{\stackrel{\rho_{*}}{\rightleftarrows}} \operatorname{Mod}\left(\mathrm{C}_{X_{s a}}\right)
$$

and we still denote by $\rho_{*}$ the restriction of $\rho_{*}$ to $\operatorname{Mod}_{\mathbb{R}-\mathrm{c}}\left(\mathrm{C}_{X}\right)$ and to $\operatorname{Mod}_{\mathrm{R}-\mathrm{c}}^{c}\left(\mathrm{C}_{X}\right)$. Recall that $\rho_{*}$ is left exact and that it induces an exact functor on $\operatorname{Mod}_{\mathbb{R}-c}\left(\mathrm{C}_{X}\right)$. Thereby we identify $F$ and $\rho_{*}(F)$. Moreover, the functor $\rho^{-1}$ is left adjoint to $\rho_{*}$ and $\rho^{-1}$ admits a left adjoint, denoted by $\rho_{!}$. Recall that $\rho_{!} \mathrm{F}$ is the sheaf on $X_{s a}$ associated to the presheaf $U \rightarrow F(\bar{U})$, for $U \in \mathrm{Op}\left(X_{s a}\right)$.

Proposition 2.1. Let $\left\{F_{i}\right\}_{i \in I}$ be a filtrant inductive system in $\operatorname{Mod}\left(C_{X_{s a}}\right)$ and let $U$ be a relatively compact subanalytic open subset of $X$. Then:

$$
\underset{i \in I}{\lim } \Gamma\left(U ; F_{i}\right) \stackrel{\sim}{\longrightarrow} \Gamma\left(U ; \underset{i \in I}{\lim } F_{i}\right) .
$$
Proposition 2.2. Let $F=\underset{i \in I}{\lim } F_{i}$ with $F_{i} \in \operatorname{Mod}\left(\mathrm{C}_{X_{s a}}\right)$ and let
$G \in D_{\mathbb{R}-c}^{b}\left(\mathrm{C}_{X}\right)$. One has:

$$
R^{k} \mathcal{H o m}(G, F) \simeq \underset{i \in I}{\lim } R^{k} \mathcal{H o m}\left(G, F_{i}\right)
$$

for each $k \in Z$.

Recall that the functor $\rho_{*}$ does not commute with direct sums in general. However, this is true when considering a direct sum of copies of a same $\mathbb{R}$-constructible module, which will suffice for our purposes.

More precisely, noting, for a set of indexes $I, F^{\oplus I}:=\oplus_{i \in I} F_{i}$ with $F_{i}=F$, we have the following property:

Lemma 2.3. Let $F$ be an object of $\operatorname{Mod}_{\mathbb{R}-c}\left(\mathbb{C}_{X}\right)$. Then for any set I of indexes one has:

$$
\rho_{*}\left(F^{\oplus I}\right) \simeq\left(\rho_{*} F\right)^{\oplus I}
$$

in $\operatorname{Mod}_{X_{s a}}\left(\mathrm{C}_{X}\right)$. 
Proof. Every object $F \in \operatorname{Mod}_{\mathbb{R}-c}\left(C_{X}\right)$ admits a finite resolution of the form:

$$
0 \rightarrow \underset{i \in I_{1}}{\oplus} \mathrm{C}_{U_{1, i}} \rightarrow \cdots \rightarrow \underset{i \in I_{m}}{\oplus} \mathrm{C}_{U_{m, i}} \rightarrow 0,
$$

by locally finite families $\left\{U_{k, i_{k}}\right\}_{k, i_{k}}$ of relatively compact open subanalytic sets of $X$ (see Appendix of [7], Theorem A.11). Since the functor $(\cdot)^{\oplus I}$ is exact on $\operatorname{Mod}\left(\mathbb{C}_{X}\right)$, we have a quasi-isomorphism:

$$
F^{\oplus I} \underset{\text { qis }}{\simeq} 0 \rightarrow \underset{i \in I_{1}}{\oplus}\left(\mathrm{C}_{U_{1, i}}\right)^{\oplus I} \rightarrow \cdots \rightarrow \underset{i \in I_{m}}{\oplus}\left(\mathbb{C}_{U_{m, i}}\right)^{\oplus I} \rightarrow 0
$$

in $\operatorname{Mod}\left(C_{X}\right)$. On the other hand, $\mathbb{R}$-constructible sheaves are injective with respect to the functor $\rho_{*}$, which entails a quasi-isomorphism

$$
\rho_{*} F \underset{\text { qis }}{\simeq} 0 \rightarrow \underset{i \in I_{1}}{\oplus} \rho_{*} \mathrm{C}_{U_{1, i}} \rightarrow \cdots \rightarrow \underset{i \in I_{m}}{\oplus} \rho_{*} \mathrm{C}_{U_{m, i}} \rightarrow 0,
$$

in $\operatorname{Mod}\left(\mathrm{C}_{X_{s a}}\right)$, hence a quasi-isomorphism

$$
\left(\rho_{*} F\right)^{\oplus I} \underset{q i s}{\simeq} 0 \rightarrow \underset{i \in I_{1}}{\oplus}\left(\rho_{*} \mathrm{C}_{U_{1, i}}\right)^{\oplus I} \rightarrow \cdots \rightarrow \underset{i \in I_{m}}{\oplus}\left(\rho_{*} \mathrm{C}_{U_{m, i}}\right)^{\oplus I} \rightarrow 0
$$

in $\operatorname{Mod}\left(\mathrm{C}_{X_{s a}}\right)$. Since weakly $\mathbb{R}$-constructible sheaves are also injective with respect to the functor $\rho_{*}$, we obtain a quasi-isomorphism

$$
\rho_{*}\left(F^{\oplus I}\right) \underset{\text { qis }}{\simeq} 0 \rightarrow \underset{i \in I_{1}}{\oplus} \rho_{*}\left(\left(\mathrm{C}_{U_{1, i}}\right)^{\oplus I}\right) \rightarrow \cdots \rightarrow \underset{i \in I_{m}}{\oplus} \rho_{*}\left(\left(\mathrm{C}_{U_{m, i}}\right)^{\oplus I}\right) \rightarrow 0
$$

in $\operatorname{Mod}\left(\mathbb{C}_{X_{s a}}\right)$. Therefore, we are reduced to prove that $\rho_{*}\left(\mathbb{C}_{U}^{\oplus I}\right) \simeq\left(\rho_{*} \mathbb{C}_{U}\right)^{\oplus I}$, for any relatively compact open subanalytic subset $U \subset X$. This will follow if we prove that, for each relatively compact open subanalytic subset $V \subset X$, there exists a finite covering $\left\{V_{i}\right\}_{i}$ of $V$ by open subanalytic sets $V_{i}$ such that $\Gamma\left(V_{i} ; \rho_{*}\left(\mathbb{C}_{U}^{\oplus I}\right)\right) \simeq \Gamma\left(V_{i} ;\left(\rho_{*} \mathrm{C}_{U}\right)^{\oplus I}\right)$.

Recall that direct sums are a particular case of inductive limits, more precisely, they are inductive limits of finite direct sums. Hence, by Proposition 2.1 we have, for any relatively compact $\Omega \in \mathrm{Op}\left(X_{s a}\right)$ :

$$
\Gamma\left(\Omega ;\left(\rho_{*} \mathrm{C}_{U}\right)^{\oplus I}\right) \simeq \Gamma\left(\Omega ; \mathrm{C}_{U}\right)^{\oplus I},
$$

since $\rho_{*}$ commutes with finite direct sums. Therefore, by the isomorphism $\mathrm{C}_{U}^{\oplus I} \simeq\left(\mathbb{C}^{\oplus I}\right)_{U}$, we have to prove that there exists a finite covering $\left\{V_{i}\right\}_{i}$ of $V$ by open subanalytic sets $V_{i}$

$$
\Gamma\left(V_{i} ;\left(\mathbb{C}^{\oplus I}\right)_{U}\right) \simeq \Gamma\left(V_{i} ; \mathbb{C}_{U}\right)^{\oplus I} .
$$

In the rest of the proof we shall use $K$ to denote either $\mathrm{C}$ or $\mathrm{C}^{\oplus I}$ and we follow the notations of [6] for constructibility on a simplicial complex. 
Let us consider the subanalytic stratification of $X$ given by

$$
(U \cap V) \sqcup(U \backslash V) \sqcup(V \backslash U) \sqcup(X \backslash(U \cup V)) .
$$

By the triangulation theorem (cf [6]) there exist a simplicial complex $(S, \Delta)$ and a homeomorphism $i:|S| \rightarrow X$ compatible with the stratification above such that $V$ is a finite union of connected open subanalytic sets of the form $i(U(\sigma))=i\left(\bigcup_{\tau \in \Delta, \tau \supseteq \sigma}|\tau|\right)$. More precisely, $V=\bigcup_{i(|\sigma|) \subset V} i(U(\sigma))$. On the other hand, given $\sigma \in \Delta$ such that $i(|\sigma|) \subset V$, and $x \in|\sigma|$, by Proposition 8.1.4 of [6] we get:

$$
\Gamma\left(i(U(\sigma)) ; K_{U}\right) \simeq \Gamma\left(U(\sigma) ; i^{-1} K_{U}\right) \simeq\left(i^{-1} K_{U}\right)_{x} \simeq i^{-1}\left(\left(K_{U}\right)_{i(x)}\right),
$$

since $i^{-1} K_{U}$ is a weakly $S$-constructible sheaf. Therefore

$$
\Gamma\left(i(U(\sigma)) ; K_{U}\right) \simeq\left\{\begin{array}{ll}
K, & \text { if } i(|\sigma|) \subset U \\
0, & \text { if } i(|\sigma|) \nsubseteq U
\end{array},\right.
$$

which entails the desired isomorphism, taking as $\left\{V_{i}\right\}_{i}$ the covering $\{i(U(\sigma))\}_{i(|\sigma|) \subset V}$ of $V$.

q.e.d.

We shall now give a short overview on the Whitney functor ([7]), and on the tempered cohomology functor, (introduced in [4] and detailedly studied in [7]).

The Whitney functor, denoted by $(\cdot) \stackrel{\mathrm{w}}{\otimes} \mathcal{C}_{X}^{\infty}$, is a functor from $D_{\mathrm{R}-c}^{b}\left(\mathrm{C}_{X}\right)$ to $D^{b}\left(\mathcal{D}_{X}\right)$, inducing an exact functor from $\operatorname{Mod}_{\mathbb{R}-c}\left(\mathbb{C}_{X}\right)$ to $\operatorname{Mod}\left(\mathcal{D}_{X}\right)$, and such that, for $U$ open subanalytic in $X$,

$$
\mathrm{C}_{U} \stackrel{\mathrm{w}}{\otimes} \mathcal{C}_{X}^{\infty}=\mathcal{I}_{X, X \backslash U}^{\infty},
$$

the sheaf of $\mathcal{C}^{\infty}$ functions on $X$ vanishing up to infinite order on $X \backslash U$.

If $\mathcal{L}$ is a $\mathcal{A}_{X}$-locally free module of finite rank, one sets

$$
F \stackrel{\mathrm{w}}{\otimes}\left(\mathcal{C}_{X}^{\infty} \underset{\mathcal{A}_{X}}{\otimes} \mathcal{L}\right):=\left(F \stackrel{\mathrm{w}}{\otimes} \mathcal{C}_{X}^{\infty}\right) \underset{\mathcal{A}_{X}}{\otimes} \mathcal{L}
$$

The tempered distribution cohomology functor, denoted by $t \mathcal{H o m}\left(\cdot, \mathcal{D} b_{X}\right)$, is a functor from $D_{\mathbb{R}-c}^{b}\left(\mathbb{C}_{X}\right)$ to $D^{b}\left(\mathcal{D}_{X}\right)$, inducing an exact functor from $\operatorname{Mod}_{\mathbb{R}-c}\left(C_{X}\right)$ to $\operatorname{Mod}\left(\mathcal{D}_{X}\right)$ and such that, for $Z$ closed subanalytic in $X$,

$$
\text { tHom }\left(\mathrm{C}_{Z}, \mathcal{D} b_{X}\right)=\Gamma_{Z}\left(\mathcal{D} b_{X}\right),
$$

the sheaf of Schwartz distributions supported by $Z$.

One notes $\operatorname{tHom}\left(\cdot, \mathcal{D} b_{X}^{v}\right):=\operatorname{tHom}\left(\cdot, \mathcal{D} b_{X}\right) \underset{\mathcal{A}_{X}}{\otimes} \mathcal{A}_{X}^{v}$. 
These two functors are extended as sheaves on the subanalytic site $X_{s a}$ ([8]) as follows:

Let $\mathcal{C}_{X_{s a}}^{\infty, \mathrm{w}}$ denote the sheaf on $X_{s a}$ of Whitney $\mathcal{C}^{\infty}$-functions, that is, the sheaf defined by:

$$
U \mapsto \Gamma\left(X ; R \mathcal{H} \operatorname{Hom}\left(\mathrm{C}_{U}, \mathrm{C}_{M}\right) \stackrel{\mathrm{w}}{\otimes} \mathcal{C}_{X}^{\infty}\right)
$$

Let $\mathcal{D} b_{X_{s a}}^{t}$ denote the sheaf on $X_{s a}$ of tempered distributions, that is, the sheaf defined by:

$$
U \mapsto \Gamma\left(X ; \operatorname{tHom}\left(\mathrm{C}_{U}, \mathcal{D} b_{X}\right)\right) .
$$

We have the following isomorphisms in $D^{b}\left(\mathcal{D}_{X}\right)$ :

For $F \in D_{\mathbb{R}-c}^{b}\left(\mathbb{C}_{X}\right)$,

$$
F \stackrel{\mathrm{w}}{\otimes} \mathcal{C}_{X}^{\infty} \simeq \rho^{-1}\left(R \mathcal{H o m}\left(D^{\prime}(F), \mathcal{C}_{X_{s a}, \mathrm{w}}^{\infty}\right)\right)
$$

and

$$
t \mathcal{H o m}\left(F, \mathcal{D} b_{X}\right) \simeq \rho^{-1}\left(R \mathcal{H o m}\left(F, \mathcal{D} b_{X_{s a}}^{t}\right)\right) .
$$

For a complex analytic manifold $X$, we denote by $\mathcal{D}_{X}$ the sheaf of differential operators of finite order, by $\mathcal{O}_{X}$ the sheaf of holomorphic functions and by $\Omega_{X}$ the sheaf of holomorphic differential forms of maximal degree. Considering the complex conjugate structure in $X$ and denoting it by $\bar{X}$, one defines the following sheaves on $X_{s a}$ :

The sheaf of holomorphic Whitney functions, $\mathcal{O}_{X_{s a}}^{\mathrm{W}}$, given by

$$
\mathcal{O}_{X_{s a}}^{\mathrm{W}}=R \mathcal{H o m}_{\rho_{!} \mathcal{D}_{\bar{X}}}\left(\rho_{!} \mathcal{O}_{\bar{X}}, \mathcal{C}_{\bar{X}_{s a}}^{\infty, \mathrm{w}}\right) .
$$

The sheaf of tempered holomorphic functions, $\mathcal{O}_{X_{s a}}^{t}$, given by

$$
\mathcal{O}_{X_{s a}}^{t}=R \mathcal{H o m} \rho_{\rho_{1} \mathcal{D}_{\bar{X}}}\left(\rho_{!} \mathcal{O}_{\bar{X}}, \mathcal{D} b_{\bar{X}_{s a}}^{t}\right) .
$$

2.2 - Review on formal extensions and the formal tempered cohomology functor

Let $\mathcal{R}$ be a $Z[\hbar]$-algebra such that $\hbar: \mathcal{R} \rightarrow \mathcal{R}$ is injective (i.e, $\mathcal{R}$ is free of $\hbar$-torsion.) We note $\mathcal{R}^{\text {loc }}:=Z\left[\hbar, \hbar^{-1}\right] \otimes_{\mathrm{Z}[\hbar]} \mathcal{R}$ and $\mathcal{R}_{0}:=\mathcal{R} / \hbar \mathcal{R}$. We obtain the functors

$$
(\cdot)^{\text {loc }}: \operatorname{Mod}(\mathcal{R}) \rightarrow \operatorname{Mod}\left(\mathcal{R}^{\text {loc }}\right), \mathcal{M} \mapsto \mathcal{M}^{\text {loc }}:=\mathcal{R}^{\text {loc }} \otimes_{\mathcal{R}} \mathcal{M},
$$

which is exact, and

$$
g r_{\hbar}: D(\mathcal{R}) \rightarrow D\left(\mathcal{R}_{0}\right), \mathcal{M} \mapsto g r_{\hbar}(\mathcal{M})=\mathcal{R}_{0} \stackrel{L}{\otimes_{\mathcal{R}} \mathcal{M}}
$$


Recall that $\mathcal{M} \in D(\mathcal{R})$ is cohomologically $\hbar$-complete if

$$
R \mathcal{H o m}{ }_{\mathcal{R}}\left(\mathcal{R}^{\text {loc }}, \mathcal{M}\right)=0 .
$$
We say that a $Z[\hbar]$-module $\mathcal{M}$ is $\hbar$-complete if $\mathcal{M} \rightarrow \lim _{\tilde{j} \geq 0} \mathcal{M} / \hbar^{j} \mathcal{M}$ is an
isomorphism.

Proposition 2.4. The functor $g r_{\hbar}$ is conservative on the category of cohomologically $\hbar$-complete objects, that is, if $\mathcal{M} \in D(\mathcal{R})$ is cohomologically $\hbar$-complete and $g r_{\hbar}(\mathcal{M})=0$, then $\mathcal{M}=0$.

Proposition 2.5. For a given cohomologically $\hbar$-complete object $\mathcal{M} \in D(\mathcal{R})$, for any $\mathcal{N} \in D(\mathcal{R}), R_{\mathcal{H}} \operatorname{lom}_{\mathcal{R}}(\mathcal{N}, \mathcal{M})$ is cohomologically $\hbar$ complete.

We set $C^{\hbar}$ to shortly denote the ring $C[[\hbar]]$ of formal power series in the $\hbar$ variable and set $\left.\mathbb{C}^{\hbar, l o c}:=\mathbb{C}((\hbar)) \simeq \mathbb{C}\left[\hbar^{-1}, \hbar\right]\right]$ the field of fractions of $\mathbb{C}^{\hbar}$.

Recall the (left exact) functor of formal extension $(\cdot)^{\hbar}: \operatorname{Mod}\left(C_{X}\right) \rightarrow$ $\operatorname{Mod}\left(\mathrm{C}_{X}^{\hbar}\right)$, defined by

$$
F \rightarrow F^{\hbar}:=\lim _{\tilde{j} \geq 0}\left(F \otimes \mathbb{C}_{X}^{\hbar} / \hbar^{j} \mathbb{C}_{X}^{\hbar}\right)
$$

We denote by $(\cdot)^{R \hbar}$ its right derived functor.

Proposition 2.6. For any $F \in D^{b}\left(\mathrm{C}_{X}\right)$, its formal extension $F^{R \hbar}$ is cohomologically $\hbar$-complete.

Proposition 2.7. Let $\mathcal{I}$ be either a basis of open subsets of a site $X$ or, assuming that $X$ is a locally compact topological space, a basis of compact subsets. Denote by $\mathcal{J}_{\mathcal{I}}$ the full subcategory of $\operatorname{Mod}\left(\mathrm{C}_{X}\right)$ consisting of $\mathcal{I}$ acyclic objects, i.e., sheaves $\mathcal{N}$ for which $H^{k}(S ; \mathcal{N})=0$ for all $k>0$ and all $S \in \mathcal{I}$. Then $\mathcal{J}_{\mathcal{I}}$ is injective with respect to the functor $(\cdot)^{\hbar}$. In particular, for $\mathcal{N} \in \mathcal{J}_{\mathcal{I}}$, we have $\mathcal{N}^{\hbar} \simeq \mathcal{N}^{R \hbar}$.

The following result is contained in [1], Lemma 2.3:

Lemma 2.8. Assume that $\mathcal{R}$ is a $\mathrm{C}_{X}$-algebra. Then, for $\mathcal{M}, \mathcal{N} \in D^{b}(\mathcal{R})$, we have an isomorphism in $D^{b}\left(\mathrm{C}_{X}^{\hbar}\right)$

$$
R \mathcal{H o m}{ }_{\mathcal{R}}(\mathcal{M}, \mathcal{N})^{R \hbar} \simeq R \mathcal{H o m}{ }_{\mathcal{R}}\left(\mathcal{M}, \mathcal{N}^{R \hbar}\right) .
$$


We recall the properties of the functor $(\cdot)^{R \hbar}$ proved in [1] in view of the subanalytic site.

LEMma 2.9. (i) The functors $\rho^{-1}$ and $(\cdot)^{R \hbar}$ commute, that $i s$, for $G \in D^{b}\left(\mathbb{C}_{X_{s a}}\right)$ we have $\left(\rho^{-1} G\right)^{R \hbar} \simeq \rho^{-1}\left(G^{R \hbar}\right)$ in $D^{b}\left(\mathbb{C}_{X}^{\hbar}\right)$.

(ii) The functors $R \rho_{*}$ and $(\cdot)^{R \hbar}$ commute, that is, for $F \in D^{b}\left(\mathrm{C}_{X}\right)$ we have $\left(R \rho_{*} F\right)^{R \hbar} \simeq R \rho_{*}\left(F^{R \hbar}\right)$ in $D^{b}\left(\mathrm{C}_{X_{s a}}^{\hbar}\right)$.

Lemma 2.10. Given $F \in D_{\mathbb{R}-c}^{b}\left(\mathbb{C}_{X}^{\hbar}\right), F$ is isomorphic to a complex:

$$
0 \rightarrow \underset{i \in I_{1}}{\oplus} \mathbb{C}_{U_{1, i}}^{\hbar} \rightarrow \cdots \rightarrow \underset{i \in I_{m}}{\oplus} \mathbb{C}_{U_{m, i}}^{\hbar} \rightarrow 0,
$$

for locally finite families $\left\{U_{j, i}\right\}_{j, i}$ of relatively compact subanalytic open subsets of $X$.

Lemma 2.11. For $F \in D_{\mathbb{R}-c}^{b}\left(\mathrm{C}_{X}\right)$, we have $F^{R \hbar} \simeq F^{\hbar} \simeq \mathrm{C}_{X}^{\hbar} \otimes F$.

Recall that a resolution of $F$ as in Lemma 2.10 is called an "almost free" resolution.

The sheaves $\mathcal{C}_{X_{s a}, t, \hbar}^{\infty}, \mathcal{D} b_{X_{s a}}^{t, \hbar}$ and $\mathcal{O}_{X_{s a}}^{t, \hbar}$ on $X_{s a}$ were studied in [1], and proved to be cohomologically $\hbar$-complete. The authors also introduced the formal extension of $t \mathcal{H o m}\left(\cdot, \mathcal{O}_{X}\right)$, the functor of tempered holomorphic cohomology, and noted it $T H_{\hbar}(\cdot): D_{\mathrm{R}-c}^{b}\left(\mathrm{C}_{X}^{\hbar}\right) \rightarrow D^{b}\left(\mathcal{D}_{X}\right)$ by setting

$$
F \rightarrow T H_{\hbar}(F):=\rho^{-1} R \mathcal{H} \operatorname{lom}_{\mathbb{C}_{X_{s a}}^{\hbar}}\left(\rho_{*} F, \mathcal{O}_{X_{s a}}^{t, \hbar}\right),
$$

hence, for any $F \in D_{\mathbb{R}-c}^{b}\left(C_{X}^{\hbar}\right), T H_{\hbar}(F)$ is cohomologically $\hbar$-complete.

\section{Topological Duality}

Although the literature on the subject is wide, for the reader convenience we restrict our references to [2] and to [11] for the topological notions we need in the sequel. Recall that we say that a (real or complex) topological vector space is of type $\mathrm{FN}$ if it is Fréchet nuclear and we use the notation DFN for the strong dual of a Fréchet nuclear space (which is still FN).

As proved, for instance in 2. of [11], the strong topological dual of a (countable) projective limit of $\mathrm{FN}$ spaces, which is still a $\mathrm{FN}$ space, is topologically isomorphic to the inductive limit of the correspondent (strong) dual spaces, so it is still a DFN space.

We set $\mathbb{C}^{[\hbar]}:=\mathbb{C}((\hbar)) / \mathbb{C}^{\hbar}$. 
For $j \in Z_{>0}$, let us note by $\mathrm{C}_{j}^{[\hbar]}$ the image of $\mathbb{C}^{\hbar}+\mathrm{C}\left[\hbar^{-1}\right]_{j}$ in the quotient $\mathrm{C}((\hbar)) / \mathbb{C}_{X}^{\hbar}$, where $\mathbb{C}\left[\hbar^{-1}\right]_{j}$ denotes the set of complex polynomials of degree at most $j$ in the $\hbar^{-1}$ variable, in other words, $\mathbb{C}_{j}^{[\hbar]}=\left(\hbar^{-j} \mathbb{C}^{\hbar}\right) / \mathbb{C}^{\hbar}$. Hence, as a C-vector space, $\mathbb{C}^{[\hbar]}$ is isomorphic to the polynomial ring $\mathrm{C}\left[\hbar^{-1}\right]$. However, as a quotient of $\mathrm{C}((\hbar)), \mathbb{C}^{[\hbar]}$ is not a ring and we shall keep in mind its $\mathbb{C}^{\hbar}$-module structure.

Observe that $\mathbb{C}^{\hbar} \simeq \lim _{n>0} \mathbb{C}^{\hbar} / \hbar^{n} \mathbb{C}^{\hbar}$ is topologically isomorphic to the product of the $\mathrm{FN}$ topological $\mathrm{C}$-vector spaces $\mathrm{C} \hbar^{n-1}$ and hence it is a FN space. On the other side, $\mathbb{C}^{[\hbar]} \simeq \underset{n>0}{\lim } \mathbb{C}_{n}^{[\hbar]}$ is topologically an inductive limit of DFN spaces so it is a DFN space. Moreover, for each $n, \mathrm{C}^{\hbar} / \hbar^{n} \mathrm{C}^{\hbar}$ and $\mathbb{C}_{n}^{[\hbar]}$ are mutually dual. So $\mathbb{C}^{\hbar}$ and $\mathbb{C}^{[\hbar]}$ are mutually dual. Indeed, it is well known that the obtained duality is perfect and given by $\langle f, g\rangle=R e s_{\hbar=0}(f g)$.

Thus, given a $\mathrm{FN}$ topological C-vector space $V$, the space $V^{\hbar} \simeq$ $\lim V \otimes \mathrm{C}^{\hbar} / \hbar^{n} \mathrm{C}^{\hbar}$ is the topological projective limit of the $\mathrm{FN}$ spaces $\overleftarrow{n>0}$

$V \otimes \mathrm{C}^{\hbar} / \hbar^{n} \mathrm{C}^{\hbar}$ (a tensor product of $\mathrm{FN}$ spaces), so it is still FN. In addition, noting $V^{*}$ its strong topological dual and observing that, for each $n$, the topological dual of $V \otimes \mathrm{C}^{\hbar} / \hbar^{n} \mathrm{C}^{\hbar}$ is $V^{*} \otimes \mathrm{C}_{n}^{[\hbar]}$ (a DFN space), we deduce that $V^{\hbar}$ and $V^{*} \otimes \mathbb{C}^{[\hbar]}$ are topologically dual to each other, the last one being a DFN space.

We shall say that two complexes $V^{\bullet}$ and $W^{\bullet}$ of topological vector spaces of type FN and DFN, respectively, are dual to each other if each entry $W^{-i}$ of $W^{\bullet}$ is the topological dual of the entry $V^{i}$ of $V^{\bullet}$ and the morphism $w^{i}: W^{-i-1} \rightarrow W^{-i}$ is the transpose of $v^{i}: V^{i} \rightarrow V^{i+1}$.

\section{Formal extension of the Whitney functor}

Let $X$ be a real analytic manifold.

Definition 4.1. The sheaf of formal Whitney $\mathcal{C}_{X}^{\infty}$ functions is the object of $\operatorname{Mod}\left(\mathbb{C}_{X_{s a}}^{\hbar}\right)$ given by

$$
\mathcal{C}_{X_{s a}, \mathrm{w}, \hbar}^{\infty}:=\left(\mathcal{C}_{X_{s a}}^{\infty, \mathrm{w}}\right)^{\hbar} .
$$

Recall that $R \Gamma\left(U ; \mathcal{C}_{X_{s a}, \mathrm{w}}\right) \simeq R \Gamma\left(X ; D^{\prime} \mathrm{C}_{U} \stackrel{\mathrm{w}}{\otimes} \mathcal{C}_{X}^{\infty}\right)$, for all open subanalytic subsets $U$ of $X$ (see [9]). So for those $U$ such that $D^{\prime} C_{U}$ is concentrated in degree zero (for instance the so called locally cohomologically trivial (l.c.t) subanalytic open subsets), $D^{\prime} \mathrm{C}_{U} \stackrel{\mathrm{w}}{\otimes} \mathcal{C}_{X}^{\infty}$ being a soft sheaf, $R \Gamma\left(U ; \mathcal{C}_{X_{s a}}^{\infty, \mathrm{w}}\right)$ is concentrated in degree zero. Since l.c.t. open subanalytic sets form a basis 
for the site $X_{s a}$, we have, by Proposition 2.7:

$$
\mathcal{C}_{X_{s a}, \mathrm{w}, \hbar}^{\infty} \simeq\left(\mathcal{C}_{X_{s a}, \mathrm{w}}^{\infty}\right)^{R \hbar}
$$

Therefore, since formal extensions are cohomologically $\hbar$-complete (see Proposition 2.2 of [1]), $\mathcal{C}_{X_{s a}, \mathrm{w}, \hbar}$ is cohomologically $\hbar$-complete.

Let $D_{\hbar}^{\prime}$ denote the functor $\mathrm{D}^{b}\left(\mathbb{C}_{X}^{\hbar}\right)^{o p} \rightarrow \mathrm{D}^{b}\left(\mathbb{C}_{X}^{\hbar}\right), F \mapsto \mathrm{RHom}_{\mathbb{C}_{X}^{h}}\left(F, \mathbb{C}_{X}^{\hbar}\right)$.

Definition 4.2. We define the "formal extension of Whitney functor"

$$
(\cdot)^{\mathrm{w}, \hbar} \mathcal{C}_{X}^{\infty}: D_{\mathbb{R}-c}^{b}\left(\mathbb{C}_{X}^{\hbar}\right) \rightarrow D^{b}\left(\mathbb{C}_{X}^{\hbar}\right),
$$

as the composition of derived functors

$$
F \mapsto F \stackrel{\mathrm{w}, \hbar}{\otimes} \mathcal{C}_{X}^{\infty}:=\rho^{-1} R \mathcal{H}{ }^{\infty} m_{\mathrm{C}_{X_{s a}}^{h}}\left(\rho_{*}\left(D_{\hbar}^{\prime} F\right), \mathcal{C}_{X_{s a}}^{\infty, \mathrm{w}, \hbar}\right) .
$$

Since $\mathcal{C}_{X_{s a}}^{\infty, \mathrm{w}}$ belongs to $\operatorname{Mod}\left(\rho_{!} \mathcal{D}_{X}\right)$, the functor $\rho^{-1}$ commutes with $(\cdot)^{R \hbar}$ and $\rho^{-1} \circ \rho_{!} \simeq \mathrm{id}, F \stackrel{\mathrm{w}, \hbar}{\otimes} \mathcal{C}_{X}^{\infty}$ is an object of $D^{b}\left(\mathcal{D}_{X}^{\hbar}\right)$, in other words, $(\cdot) \stackrel{\mathrm{w}, \hbar}{\otimes} \mathcal{C}_{X}^{\infty}$ is a functor from $\mathrm{D}_{\mathbb{R}-c}^{b}\left(\mathbb{C}_{X}^{\hbar}\right)$ to $D^{b}\left(\mathcal{D}_{X}^{\hbar}\right)$. Moreover, $F \stackrel{\text { w, }, \hbar}{\otimes} \mathcal{C}_{X}^{\infty}$ is cohomologically $\hbar$-complete.

Lemma 4.3. Let $F \in \operatorname{Mod}_{\mathbb{R}-c}\left(\mathrm{C}_{X}\right)$. Then

$$
F^{\hbar} \stackrel{\mathrm{w}, \hbar}{\otimes} \mathcal{C}_{X}^{\infty} \simeq\left(F \stackrel{\mathrm{w}}{\otimes} \mathcal{C}_{X}^{\infty}\right)^{\hbar}
$$

in $\operatorname{Mod}\left(\mathcal{D}_{X}^{\hbar}\right)$, hence it is concentrated in degree zero and is a soft sheaf.

Proof. The result follows from (7), Lemmas 2.8 and 2.9 and Proposition 2.7 .

q.e.d.

REMARK 4.4. Given $F \in \mathrm{D}_{\mathbb{R}-c}^{b}\left(\mathbb{C}_{X}^{\hbar}\right)$, choosing a resolution $F^{\bullet}$ of $F$ as in (6), that is, such that each entry $F^{i}$ is isomorphic to a locally finite sum of $\mathbb{C}_{X}^{\hbar}$-modules of the form $\mathbb{C}_{U}^{\hbar}$, with $U \in \mathrm{Op}\left(X_{s a}\right)$, we obtain that $F^{\mathrm{w}, \hbar} \mathcal{C}_{X}^{\infty}$ is isomorphic to a bounded complex $F^{\bullet} \stackrel{\mathrm{w}, \hbar}{\otimes} \mathcal{C}_{X}^{\infty}$ which provides a soft resolution of $F \stackrel{\text { w, },}{\otimes} \mathcal{C}_{X}^{\infty}$. Therefore, for any open subanalytic set $U$ in $X$, we have

$$
R \Gamma\left(U ; F \stackrel{\mathrm{w}, \hbar}{\otimes} \mathcal{C}_{X}^{\infty}\right) \simeq \Gamma\left(U ; F^{\bullet} \stackrel{\mathrm{w}, \hbar}{\otimes} \mathcal{C}_{X}^{\infty}\right) .
$$

When $X$ is a complex analytic manifold, denoting by $\bar{X}$ the complex conjugate manifold of $X$ and by $X^{\mathbb{R}}$ the underlying real analytic manifold identified with the diagonal of $X \times \bar{X}$, we may also define the $\hbar$-version of 
the sheaf $\mathcal{O}_{X}^{\mathrm{W}}$ on $X_{s a}$, by setting:

DEFINITION 4.5.

$$
\mathcal{O}_{X_{s a}}^{\mathrm{w}, \hbar}:=R \mathcal{H o m} \rho_{\rho !} \mathcal{D}_{\bar{X}}\left(\rho_{!} \mathcal{O}_{\bar{X}}, \mathcal{C}_{X_{s a}^{\mathrm{R}}}^{\infty, \mathrm{w}, \hbar}\right) .
$$

As a consequence of (7) together with Lemma 2.8 we have:

$$
\mathcal{O}_{X_{s a}}^{\mathrm{W}, \hbar} \simeq\left(\mathcal{O}_{X_{s a}}^{\mathrm{W}}\right)^{R \hbar}
$$

so, in particular, $g r_{\hbar}\left(\mathcal{O}_{X_{s a}}^{\mathrm{w}, \hbar}\right) \simeq \mathcal{O}_{X_{s a}}^{\mathrm{W}}$.

We now introduce the formal extension of the holomorphic Whitney functor, ( · ) $\stackrel{\mathrm{w}, \hbar}{\otimes} \mathcal{O}_{X}: \mathrm{D}_{\mathbb{R}-c}^{b}\left(\mathbb{C}_{X}^{\hbar}\right) \rightarrow \mathrm{D}^{b}\left(\mathcal{D}_{X}^{\hbar}\right)$, by setting:

$$
F \stackrel{\mathrm{w}, \hbar}{\otimes} \mathcal{O}_{X}:=\rho^{-1} R \mathcal{H o m} m_{\mathrm{C}_{X_{s a}}^{h}}\left(\rho_{*}\left(D_{\hbar}^{\prime} F\right), \mathcal{O}_{X_{s a}^{\mathrm{w}}, \hbar}^{\mathrm{w}}\right) \simeq R \mathcal{H} \operatorname{Hom}_{\rho_{!} \mathcal{D}_{\bar{X}}}\left(\rho_{!} \mathcal{O}_{\bar{X}}, F^{\mathrm{w}, \hbar} \stackrel{\mathcal{C}_{X_{s a}^{\mathrm{R}}}^{\infty}}{)}\right) .
$$

Therefore, for any $F \in D_{\mathrm{R}-c}^{b}\left(\mathrm{C}_{X}^{\hbar}\right), F \stackrel{\mathrm{w}, \hbar}{\otimes} \mathcal{O}_{X}$ is cohomologically $\hbar$-complete and $g r_{\hbar}\left(F \stackrel{\mathrm{w}, h}{\otimes} \mathcal{O}_{X}\right) \simeq g r_{h}(F) \stackrel{\mathrm{w}}{\otimes} \mathcal{O}_{X}$. Moreover, by Lemmas 2.8 and 2.9 we get

Corollary 4.6. Let $F \in D_{\mathbb{R}-c}^{b}\left(\mathrm{C}_{X}\right)$. Then

$$
F^{\hbar} \stackrel{\mathrm{w}, \hbar}{\otimes} \mathcal{O}_{X} \simeq\left(F \stackrel{\mathrm{w}}{\otimes} \mathcal{O}_{X}\right)^{R \hbar}
$$

in $D^{b}\left(\mathcal{D}_{X}^{\hbar}\right)$.

Recall that one notes by $D_{r h}^{b}\left(\mathcal{D}_{X}\right)$ the full triangulated category of $D_{\text {coh }}^{b}\left(\mathcal{D}_{X}\right)$ of the objects having regular holonomic cohomology and by $D_{r h}^{b}\left(\mathcal{D}_{X}^{\hbar}\right)$ the full triangulated subcategory of $D_{c o h}^{b}\left(\mathcal{D}_{X}^{\hbar}\right)$ of the objects $\mathcal{M}$ such that $g r_{\hbar}(\mathcal{M}) \in D_{r h}^{b}\left(\mathcal{D}_{X}\right)$.

Proposition 4.7. Let $\mathcal{M} \in D_{r h}^{b}\left(\mathcal{D}_{X}^{\hbar}\right)$ and let $F \in D_{\mathbb{R}-c}^{b}\left(\mathbb{C}_{X}\right)$. Then, the natural morphism:

$$
\left.R \mathcal{H o m}{\operatorname{\mathcal {D}_{X}^{h}}}_{(\mathcal{M}}\left(F \otimes \mathcal{O}_{X}\right)^{R \hbar}\right) \rightarrow R \mathcal{H o m} \operatorname{\mathcal {D}}_{X}^{h}\left(\mathcal{M}, F^{\hbar} \stackrel{\mathrm{w}, \hbar}{\otimes} \mathcal{O}_{X}\right)
$$

is an isomorphism in $D^{b}\left(\mathbb{C}_{X}^{\hbar}\right)$.

Proof. Since both sides of the morphism (9) are cohomologically $\hbar$ complete by Propositions 2.5 and 2.6, by Proposition 2.4 it is enough to apply $g r_{\hbar}$ and then the result follows by Corollary 4.6 and Corollary 6.2 of [7] 
which prove the isomorphism

$$
R \mathcal{H o m} \mathcal{D}_{X}\left(g r_{\hbar}(\mathcal{M}), F \otimes \mathcal{O}_{X}\right) \stackrel{\sim}{\longrightarrow} R \mathcal{H} \operatorname{Hom}_{\mathcal{D}_{X}}\left(g r_{\hbar}(\mathcal{M}), F \stackrel{\mathrm{w}}{\otimes} \mathcal{O}_{X}\right) .
$$

q.e.d.

Remark that Theorem A.9 of [7] entails the existence of almost free resolutions in the framework of coherent $\mathcal{D}_{X}^{\hbar}$-modules:

Proposition 4.8. Let $\mathcal{M} \in D_{\text {coh }}^{b}\left(\mathcal{D}_{X}^{\hbar}\right)$. Then there exist a family $\left(U_{j}\right)_{j \in J} \in \mathrm{Op}\left(X_{s a}\right)$, a complex $\mathcal{L}^{\bullet} \in D_{\text {coh }}^{b}\left(\mathcal{D}_{X}^{\hbar}\right)$ and a quasi-isomorphism $\mathcal{L}^{\bullet} \rightarrow \mathcal{M}$, such that:

(i) Each $U_{j}$ is relatively compact,

(ii) Each entry $\mathcal{L}^{i}$ of $\mathcal{L}$ is a locally finite direct sum of the form $\underset{j \in J_{i}}{\oplus} \mathcal{D}_{X U_{j}}^{\hbar}$, where $J=\bigcup_{i} J_{i}$.

\section{Algebraic extension of tempered cohomology}

\section{1 - The functor of algebraic extension}

Given a sheaf $F$ of $\mathrm{C}^{\hbar}$-modules on a topological space $X$, or more generally, a sheaf in $\operatorname{Mod}\left(C_{X}^{\hbar}\right)$, for a site $X$ (we shall not distinguish these situations, unless otherwise explicited), one sets $F^{l o c}:=\mathbb{C}_{X}((\hbar)) \otimes F$. Clearly $(\cdot)^{l o c}$ is an exact functor.

For $F \in D^{b}\left(\mathbb{C}_{X}^{\hbar}\right)$ we set $F^{l o c}:=\mathrm{C}_{X}((\hbar)) \otimes_{\mathrm{C}_{X}^{h}} F$.

Definition 5.1. Given a sheaf $F$ of $\mathrm{C}^{\hbar}$-modules, we denote by $F^{[\hbar]}$ the sheaf $F \underset{\mathrm{C}_{X}^{h}}{\otimes} \underset{X}{\left[\mathrm{C}^{[\hbar]}\right.}$.

Hence $(\cdot)^{[\hbar]}$ defines a right exact functor on $\operatorname{Mod}\left(\mathbb{C}_{X}^{\hbar}\right)$ and we note $(\cdot)^{L[\hbar]}$ its left derived functor. By construction $F^{[\hbar]}$ is a $\mathbb{C}_{X}^{\hbar}$-module with torsion and, when $F$ has no $\hbar$-torsion, $F^{[h]}$ is isomorphic to $F^{l o c} / F$. Note that $F^{[\hbar]}$ is, as a sheaf of $\mathrm{C}^{\hbar}$-modules, isomorphic to $\underset{\vec{j}}{\lim } \underset{\mathrm{C}_{X}^{h}}{\otimes} \mathrm{C}_{X}^{[\hbar]}$. We set $F_{j}^{[\hbar]}:=F \underset{\mathbb{C}_{X}^{h}}{\otimes} \underset{X}{[\hbar]}$. Hence

$$
\Gamma_{c}\left(X ; F^{[\hbar]}\right) \simeq \lim _{\vec{j}} \Gamma_{c}\left(X ; F_{j}^{[\hbar]}\right) .
$$

We also have $\left(F^{[\hbar]}\right)_{U} \simeq F_{U}^{[\hbar]}$, for any open subset $U$ of $X$. 
Clearly, for $F \in D^{b}\left(\mathrm{C}_{X}^{\hbar}\right)$ one has a quasi isomorphism

$$
F^{L[\hbar]}=F \stackrel{L}{\otimes_{\mathbb{C}_{X}^{\hbar}}} \mathbb{C}_{X}^{[\hbar]} \underset{Q I S}{\rightarrow}\left\{0 \rightarrow F \rightarrow F^{l o c} \rightarrow 0\right\} .
$$

Definition 5.2. We say that $F \in D^{b}\left(\mathbb{C}_{X}^{\hbar}\right)$ is cohomologically $\hbar$-torsion if

$$
F^{l o c}=0 .
$$

Therefore $F^{L[\hbar]}$ is obviously cohomologically $\hbar$-torsion. Indeed

$$
F \stackrel{L}{\otimes_{\mathrm{C}_{X}^{h}}} \mathrm{C}_{X}^{[\hbar]} \otimes_{\mathrm{C}_{X}^{h}} \mathrm{C}_{X}((\hbar))=0
$$

because $\mathbb{C}_{X}^{[\hbar]} \otimes_{\mathrm{C}_{X}^{\hbar}} \mathbb{C}_{X}((\hbar))=0$.

Let us note $g r_{\hbar} F:=\mathbb{C}_{X} \stackrel{L}{\otimes}_{\mathrm{C}_{X}^{\hbar}} F$. For instance, $g r_{\hbar} \mathcal{O}_{X}^{[\hbar]} \simeq \mathcal{O}_{X}$, $g r_{\hbar} \mathcal{D}_{X}^{[\hbar]} \simeq \mathcal{D}_{X}$

LEMma 5.3. Assume that $g r_{\hbar}(F)=0$ and that $F$ is cohomologically $\hbar$ torsion. Then $F=0$, in other words, the functor $g r_{\hbar}$ is conservative on the subcategory of $D^{b}\left(\mathbb{C}_{X}^{\hbar}\right)$ of cohomologically $\hbar$-torsion objects.

Proof. 1) Let us start by assuming that $F$ is concentrated in degree 0 . Then, since $\hbar$ is invertible on $F, F$ is a $\mathrm{C}_{X}\left[\hbar^{-1}\right]$-module, a fortiori a $\mathbb{C}((\hbar))$ module. It follows that $F \simeq F^{l o c}$, hence $F=0$.

2) To treat the general case, since $\mathcal{H}^{j}\left(F^{l o c}\right) \simeq \mathcal{H}^{j}(F)^{l o c}$, the result follows by 1 ) applied to $\mathcal{H}^{j}(F)$.

q.e.d.

The first two following results are clear:

LEMMA 5.4. Assume that $F$ is cohomologically $\hbar$-torsion, Then, for any $G \in D^{b}\left(\mathbb{C}_{X}^{\hbar}\right), G \stackrel{L}{\otimes_{\mathbb{C}_{X}^{\hbar}}} F$ is cohomologically $\hbar$-torsion.

Lemma 5.5. Given $\mathcal{N} \in D_{\text {coh }}^{b}\left(\left(\mathcal{D}_{X}^{\hbar}\right)^{o p}\right)$, and $F \in D^{b}\left(\mathcal{D}_{X}^{\hbar}\right)$, with $F$ cohomologically $\hbar$-torsion in $D^{b}\left(\mathbb{C}^{\hbar}\right)$, then $\mathcal{N} \stackrel{L}{\otimes}_{\mathcal{D}_{X}^{\hbar}} F$ is cohomologically $\hbar$-torsion.

Observe that for any $F \in \operatorname{Mod}_{\mathbb{R}-c}\left(C_{X}\right), F^{\hbar}$ is $\hbar$-torsion free, hence $\left((\cdot)^{\hbar}\right)^{[\hbar]}$ induces an exact functor on $\operatorname{Mod}_{\mathbb{R}-c}\left(\mathbb{C}_{X}\right)$.

For the sake of simplicity, for $(\cdot)^{\hbar}$-acyclic objects $G \in \operatorname{Mod}\left(\mathbb{C}_{X}\right)$, $\left((G)^{\hbar}\right)^{[\hbar]}$ will be denoted by $G^{[\hbar]}$. 
LEMma 5.6. Assume that $H \in D^{b}\left(\mathbb{C}_{X}\right)$ is $(\cdot)^{\hbar}$-acyclic and that $H^{\hbar}$ is a complex of $\hbar$-torsion free modules. Let $F \simeq H^{[\hbar]}$. Then, for any $G \in D_{\mathrm{R}-c}^{b}\left(\mathbb{C}_{X}^{\hbar}\right), R \mathcal{H} \operatorname{om}_{\mathbb{C}_{X}^{\hbar}}(G, F)$ is cohomologically $\hbar$-torsion.

Proof. Since the statement is of local nature, we may replace $G$ by an almost free resolution $G^{\bullet}$ such that each entry $G^{i}$ is a locally finite direct sum of sheaves of the form $\mathrm{C}_{\Omega_{i j}}^{\hbar}$, for given open subanalytic sets $\Omega_{i j}$. Therefore $R \mathcal{H} \mathrm{om}_{\mathrm{C}}^{\hbar}(G, F)$ is isomorphic to $\mathcal{H o m}\left(G^{\bullet}, H\right)^{[\hbar]}$ which is cohomologically $\hbar$-torsion, and the result follows.

q.e.d.

\section{2 - Algebraic extension of tempered cohomology}

Let us now assume that $X$ is a real analytic manifold and let $X_{s a}$ the associated subanaytic site. We shall also keep the notation $(\cdot)^{L[\hbar]}$ for the corresponding functor $\mathrm{D}^{b}\left(\mathbb{C}_{X_{s a}}^{\hbar}\right) \rightarrow \mathrm{D}^{b}\left(\mathrm{C}_{X_{s a}}^{\hbar}\right)$.

Lemma 5.7. (i) For $F \in D_{\mathbb{R}-c}^{b}\left(\mathbb{C}_{X}^{\hbar}\right)$, one has $\rho_{*}\left(F^{L[\hbar]}\right) \simeq\left(\rho_{*} F\right)^{L[\hbar]}$.

(ii) The functors $\rho^{-1}$ and $(\cdot)^{[\hbar]}$ commute, more precisely, for all $F \in D^{b}\left(\mathbb{C}_{X}^{\hbar}\right)$, one has $\rho^{-1}\left(F^{L[\hbar]}\right) \simeq\left(\rho^{-1} F\right)^{L[\hbar]}$ and, for any $G \in D^{b}\left(\mathbb{C}_{X}\right)$, one has $\rho^{-1}\left(G \otimes \mathbb{C}_{X}^{[\hbar]}\right) \simeq\left(\rho^{-1} G\right) \otimes \mathbb{C}_{X}^{[\hbar]}$.

Proof. (i) By Lemma 2.10 it is enough to prove that, for any open subanalytic relatively compact set $U, \rho_{*}\left(\mathbb{C}_{U}^{[\hbar]}\right) \simeq\left(\rho_{*} \mathbb{C}_{U}^{\hbar}\right)^{[\hbar]}$. This is an immediate consequence of Lemma 2.3 and the fact that $\rho_{*}$ commutes with formal extension.

(ii) The result follows from Lemma 2.10 and the fact that the functor $\rho^{-1}$ commutes with inductive limits.

q.e.d.

We define $\mathcal{D} b_{X_{s a},[\hbar]}^{t,=}\left(\mathcal{D} b_{X_{s a}}^{t, \hbar}\right)^{[\hbar]}$.

Since $\mathcal{D} b_{X_{s a}}^{t}$ is $(\cdot)^{\hbar}$-acyclic and $\mathcal{D} b_{X_{s a}}^{t, \hbar}$ is $\hbar$-torsion free we get:

$$
\mathcal{D} b_{X_{s a}}^{t,[\hbar]} \simeq\left(\mathcal{D} b_{X_{s a}}^{t}\right)^{[\hbar]} \text {. }
$$

It is then clear that $g r_{\hbar}\left(\mathcal{D} b_{X_{s a}}^{t,[\hbar]}\right) \simeq \mathcal{D} b_{X_{s a}}^{t}$.

LEMma 5.8. For all $F \in D_{\mathbb{R}-c}^{b}\left(C_{X}\right)$ the natural morphism

$$
\rho^{-1} R \mathcal{H} \mathcal{H o m}_{\mathrm{C}_{X_{s a}}}\left(F, \mathcal{D} b_{X_{s a}}^{t,[\hbar]}\right) \rightarrow\left(\rho^{-1} R \mathcal{H} \operatorname{Hom}_{\mathrm{C}_{X_{s a}}}\left(F, \mathcal{D} b_{X_{s a}}^{t}\right)\right) \otimes \mathbb{C}_{X}^{[\hbar]}
$$


is an isomorphism in $D^{b}\left(\mathbb{C}_{X}^{\hbar}\right)$ and, if $F$ is in degree zero,

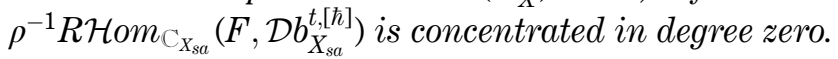

Proof. For each $k \in Z$, by Proposition 2.2, one has

$$
R^{k} \mathcal{H} o m_{C_{X_{s a}}}\left(F, \mathcal{D} b_{X_{s a}}^{t,[\hbar]}\right) \simeq R^{k} \mathcal{H} o m_{C_{X_{s a}}}\left(F, \mathcal{D} b_{X_{s a}}^{t}\right) \otimes \mathbb{C}_{X}^{[\hbar]} .
$$

Therefore, morphism (10) is an isomorphism.

Now, suppose that $F$ is in degree zero. Then $R \mathcal{H} \operatorname{Hom}_{\mathbb{C}_{X s}}\left(F, \mathcal{D} b_{X_{s a}}^{t}\right)$ is concentrated in degree 0 . Hence $R \mathcal{H} \operatorname{Hom}_{\mathrm{C}_{X_{s a}}}\left(F, \mathcal{D} b_{X_{s a}}^{t}\right) \otimes \mathrm{C}_{X}^{[\hbar]}$ is also concentrated in degree 0 . q.e.d.

DeFinition 5.9. The functor of algebraic extension of tempered distributions, noted $T D b^{[\hbar]}(\cdot)$, is the functor $\mathrm{D}_{\mathbb{R}-c}^{b}\left(\mathbb{C}_{X}^{\hbar}\right) \rightarrow D^{b}\left(\mathbb{C}_{X}^{\hbar}\right)$ defined by

$$
T D b^{[\hbar]}(F):=\rho^{-1} R \mathcal{H} \operatorname{Hom}_{\mathrm{C}_{X_{s a}}^{\hbar}}\left(\rho_{*} F, \mathcal{D} b_{X_{s a}^{t},[\hbar]}\right) .
$$

Since $\mathcal{D} b_{X_{s a}}^{t,[\hbar]}$ belongs to $\operatorname{Mod}\left(\rho_{!}\left(\mathcal{D}_{X}\right)^{\hbar}\right)$ it follows that $T D b^{[\hbar]}(F)$ is an object of $D^{b}\left(\mathcal{D}_{X}^{\hbar}\right)$. In other words, $T D b^{[\hbar]}(\cdot)$ is a functor from $\mathrm{D}_{\mathbb{R}-c}^{b}\left(\mathbb{C}_{X}^{\hbar}\right)$ to $D^{b}\left(\mathcal{D}_{X}^{\hbar}\right)$.

By Lemma 5.6, $T D b^{[\hbar]}(F)$ is cohomologically $\hbar$-torsion.

Remark 5.10. Let $F \in D_{\mathrm{R}-c}^{b}\left(\mathrm{C}_{X}\right)$. Since

$$
\rho^{-1} R \mathcal{H o m}\left(F, \mathcal{D} b_{X_{s a}}^{t}\right) \simeq t \mathcal{H o m}\left(F, \mathcal{D} b_{X}\right),
$$

Lemma 5.8 says nothing more than $T D b^{[\hbar]}\left(F^{\hbar}\right)$ is isomorphic to $t \mathcal{H o m}\left(F, \mathcal{D} b_{X}\right) \otimes \mathbb{C}_{X}^{[\hbar]}$. Moreover the isomorphism is compatible with the structure of $\mathcal{D}_{X}^{\hbar}$-modules.

In particular, since $t \mathcal{H o m}\left(\mathbb{C}_{U}, \mathcal{D} b_{X}\right) \otimes \mathbb{C}_{X}^{[\hbar]}$, being a $\mathcal{C}_{X}^{\infty}$-module, is a soft sheaf, we have that $R \Gamma\left(U ; \mathcal{D} b_{X_{s a}}^{t,[\hbar]}\right) \simeq R \Gamma\left(X ; \operatorname{tHom}\left(\mathbb{C}_{U}, \mathcal{D} b_{X}\right) \otimes \mathbb{C}_{X}^{[\hbar]}\right)$ is concentrated in degree zero. Namely, if $U$ is relatively compact,

$$
\Gamma\left(U ; \mathcal{D} b_{X_{s a}}^{t,[\hbar]}\right) \simeq \Gamma\left(U ; \mathcal{D} b_{X_{s a}}^{t}\right) \otimes \mathbb{C}_{X}^{[\hbar]}
$$

by Proposition 2.1 .

REMARK 5.11. For any $F \in \mathrm{D}_{\mathrm{R}-c}^{b}\left(\mathbb{C}_{X}^{\hbar}\right)$, choosing a resolution of $F$ as in (6), we conclude that $T D b^{[\hbar]}(F)$ is isomorphic to a bounded complex $T D b^{[\hbar]}\left(F^{\bullet}\right)$ such that each entry is isomorphic to a locally finite sum of $\mathcal{C}_{X}^{\infty}$ modules of the form $T D b^{[\hbar]}\left(\mathbb{C}_{U}^{\hbar}\right)$, with open subanalytic sets $U$. This provides a soft resolution of $T D b^{[\hbar]}(F)$. Namely, for any open subanalytic set $U$ 
in $X$, we have

$$
R \Gamma_{c}\left(U ; T D b^{[\hbar]}(F)\right) \simeq \Gamma_{c}\left(U ; T D b^{[\hbar]}\left(F^{\bullet}\right)\right)
$$

and

$$
R \Gamma\left(U ; T D b^{[\hbar]}(F)\right) \simeq \Gamma\left(U ; T D b^{[\hbar]}\left(F^{\bullet}\right)\right) .
$$

We obtain a functor of algebraic extension of tempered holomorphic functions

$$
T H^{[\hbar]}(\cdot): \mathrm{D}_{\mathbb{R}-c}^{b}\left(\mathbb{C}_{X}^{\hbar}\right) \rightarrow \mathrm{D}^{b}\left(\mathcal{D}_{X}^{\hbar}\right)
$$

by setting

$$
F \mapsto \rho^{-1} R \mathcal{H o m} \mathrm{C}_{\mathrm{C}_{s a}^{h}}\left(\rho_{*} F, \mathcal{O}_{X_{s a}}^{t,[\hbar]}\right)
$$

Clearly, we have, for $F \in D_{\mathbb{R}-c}^{b}\left(\mathbb{C}_{X}^{\hbar}\right)$,

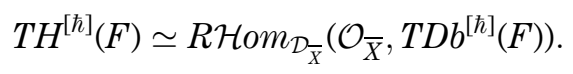

In particular, $T H^{[\hbar]}(F)$ is cohomologically $\hbar$-torsion. Moreover, it is also clear that $g r_{\hbar}\left(T H^{[\hbar]}(F)\right) \simeq t \mathcal{H o m}\left(g r_{\hbar}(F), \mathcal{O}_{X}\right)$.

Therefore, $T D b^{[\hbar]}(\cdot)$ can be understood as the algebraic extension of the functor $t \mathcal{H o m}\left(\cdot ; \mathcal{D} b_{X}\right)$ and $T H^{[\hbar]}(\cdot)$ can be understood as the algebraic extension of the functor $t \mathcal{H o m}\left(\cdot ; \mathcal{O}_{X}\right)$.

REMARK 5.12. Let $F \in D_{\mathrm{R}-c}^{b}\left(\mathrm{C}_{X}\right)$. Since one has

$$
\rho^{-1} R \mathcal{H o m}\left(F, \mathcal{O}_{X_{s a}}^{t}\right) \simeq \operatorname{tHom}\left(F, \mathcal{O}_{X}\right),
$$

it follows that $T H^{[\hbar]}\left(F^{\hbar}\right)$ is isomorphic to $\operatorname{tHom}\left(F, \mathcal{O}_{X}\right) \otimes \mathrm{C}_{X}^{[\hbar]}$. In particular, if $U$ is an open Stein subanalytic relatively compact set, and $X$ is a Stein manifold

$$
R \Gamma\left(U ; \mathcal{O}_{X_{s a}}^{t,[\hbar]}\right) \simeq R \Gamma\left(X ; T H^{[\hbar]}\left(\mathrm{C}_{U}^{\hbar}\right)\right)
$$

is concentrated in degree 0 .

All these isomorphisms are compatible with the structure of $\mathcal{D}_{X}^{\hbar}$-modules.

As an application we obtain:

Proposition 5.13. Let $\mathcal{M} \in D_{r h}^{b}\left(\mathcal{D}_{X}^{\hbar}\right)$ and let $F \in D_{\mathrm{R}-c}^{b}\left(\mathbb{C}_{X}^{\hbar}\right)$. Then, the natural morphism:

$$
R \mathcal{H o m}{\mathcal{\mathcal { D } _ { X } ^ { h }}}\left(\mathcal{M}, T H^{[\hbar]}(F)\right) \rightarrow R \mathcal{H} \operatorname{Hom}_{\mathcal{D}_{X}^{h}}\left(\mathcal{M}, R \mathcal{H o m} m_{\mathrm{C}_{X}^{h}}\left(F, \mathcal{O}_{X}^{[\hbar]}\right)\right)
$$

is an isomorphism in $D^{b}\left(\mathrm{C}_{X}^{\hbar}\right)$. 
Proof. Recall that $T H^{[\hbar]}(F)$ is cohomologically $\hbar$-torsion. Using Lemma 5.6 with $H=\mathcal{O}_{X}$, we see that $R \mathcal{H}$ om $_{\mathrm{C}_{X}^{\hbar h}}\left(F, \mathcal{O}_{X}^{[\hbar]}\right)$ is also cohomologically $\hbar$-torsion.

Thus Lemma 5.5 entails that both sides of (12) are cohomologically $\hbar$ torsion. The proof then follows by Lemma 5.3 and the isomorphism

$R \mathcal{H o m}{\mathcal{\mathcal { D } _ { X }}}\left(\operatorname{gr}(\mathcal{M}), \operatorname{tHom}\left(\operatorname{gr}(F), \mathcal{O}_{X}\right)\right) \stackrel{\sim}{\longrightarrow} R \mathcal{H} \operatorname{Hom}_{\mathcal{D}_{X}}\left(g r_{\hbar}(\mathcal{M}), R \mathcal{H o m}\left(g r_{\hbar}(F), \mathcal{O}_{X}\right)\right)$ proved in [4].

q.e.d.

To end this section, we remark that our perspective here is to work in the framework of $\mathbb{C}^{\hbar}$-algebras, or sheaves of modules over such algebras. However, the algebraic extensions of $\mathcal{O}_{X}$ or of $\mathcal{D}_{X}$ as well the associated categories of modules, have their own interest, but we shall not develop here such theory.

Let us just remark some obvious facts. $\mathcal{D}_{X}^{[\hbar]}$ is a flat $\mathcal{D}_{X}$-module and $\mathcal{O}_{X}^{[\hbar]}$ is a flat $\mathcal{O}_{X}$-module. We may endow $\mathcal{D}_{X}^{[\hbar]}$ with the filtration $F_{m}\left(\mathcal{D}_{X}^{[\hbar]}\right)$ image of $F_{m}\left(\mathcal{D}_{X}\right)\left[\hbar^{-1}\right]$ in $\mathcal{D}_{X}^{[\hbar]}$, where $\left(F_{m}\left(\mathcal{D}_{X}\right)\right)_{m \geq 0}$ denotes the filtration on $\mathcal{D}_{X}$ by the usual order.

\section{Duality for formal and algebraic extension functors}

We shall now state and prove our main results.

Let $X$ be a real analytic manifold. As proved in [4], Lemma 4.3, given $F \in D_{\mathrm{R}-c}^{b}\left(\mathrm{C}_{X}\right)$, for any open subanalytic set $U, \Gamma\left(U ; F \stackrel{w}{\otimes} \mathcal{C}_{X}^{\infty}\right)$ is a complex of FN spaces, $\Gamma_{c}\left(U ; \operatorname{tHom}\left(F, \mathcal{D} b_{X}^{v}\right)\right)$ is a complex of DFN spaces and they are dual to each other. Thus, by the results recalled in Section 3, $\Gamma\left(U ; \mathcal{C}_{X}^{\infty, \hbar}\right)$, as a countable product of $\mathrm{FN}$ spaces is a $F N$ topological vector space, $\Gamma_{c}\left(U ; \mathcal{D} b_{X}^{[\hbar]}\right) \simeq \Gamma_{c}\left(X ; \mathcal{D} b_{X}\right) \otimes \mathbb{C}_{X}^{[\hbar]}$, as an inductive limit of a countable family of DFN spaces is a DFN vector space, and they are dual to each other.

Let us now consider $F \in D_{\mathbb{R}-c}^{b}\left(\mathbb{C}_{X}^{\hbar}\right)$. We shall denote $T D b^{[\hbar]}(F) \otimes_{\mathcal{A}_{X}} \mathcal{A}_{X}^{v}$ by $T D b^{[\hbar]}(F)^{v}$ and denote $T H^{[\hbar]}(F) \otimes_{\mathcal{O}_{X}} \Omega_{X}$ by $T H^{[\hbar]}(F)^{v}$.

Proposition 6.1. Let $F \in D_{\mathbb{R}-c}^{b}\left(\mathbb{C}_{X}^{\hbar}\right)$. Then:

1. $R \Gamma\left(X ; F \stackrel{\mathrm{w}, \hbar}{\otimes} \mathcal{C}_{X}^{\infty}\right)$ is isomorphic to a complex of $F N$ spaces.

2. $R \Gamma_{c}\left(X ; T D b^{[\hbar]}(F)^{v}\right)$ is isomorphic to a complex of DFN spaces.

3. The complexes respectively described in (1) and in (2) can be chosen as to be dual to each other. 
Proof. 1. We shall adapt the argument of Proposition 2.2 of [7].

a) By Lemma 4.3 and Proposition 2.2 of (loc.cit), the assertion is true for $F=\mathbb{C}_{U}^{\hbar}$, with arbitrary $U$ open subanalytic, since $\Gamma\left(X ;\left(\mathbb{C}_{U} \stackrel{\mathrm{w}}{\otimes} \mathcal{C}_{X}^{\infty}\right)^{\hbar}\right) \simeq$ $\Gamma\left(X ;\left(\mathrm{C}_{U} \stackrel{\mathrm{w}}{\otimes} \mathcal{C}_{X}^{\infty}\right)\right)^{\hbar}$.

b) Now assume that $F$ has compact support. Then, by Lemma $2.10, F$ is quasi-isomorphic to a bounded complex :

$$
F^{\bullet}: \cdots \rightarrow F^{-1} \rightarrow F^{0} \rightarrow 0
$$

where $F^{0}$ is in degree 0 and each $F^{i}$ is a finite direct sum of sheaves of type $\mathbb{C}_{U}^{\hbar}$, $U$ open subanalytic relatively compact. Hence, by a), applying the functor $\Gamma\left(X ;(\cdot) \stackrel{\mathrm{w}, \hbar}{\otimes} \mathcal{C}_{X}^{\infty}\right)$ to the complex above, we obtain an isomorphism $R \Gamma\left(X ; F^{\mathrm{w}, \hbar} \stackrel{\mathcal{C}_{X}^{\infty}}{ }\right) \simeq V^{\bullet}$, where of $V^{\bullet}$ is a complex of type $\mathrm{FN}$.

c) To treat the general case, let us take an increasing sequence $\left\{Z_{n}\right\}_{n \in \mathbb{N}}$ of compact subanalytic subsets such that $X$ is the union of the interiors of the $Z_{n}$. Let us consider a resolution of $F, F^{\bullet}$, given by Lemma 2.10. For each $n$, note by $F_{Z_{n}}^{\bullet}$ the associated resolution of $F_{Z_{n}}$.

Each entry $F^{i}$ of $F^{\bullet}$ being a locally finite direct sum of the form $\oplus_{j_{i}} \mathrm{C}_{U_{j_{i}}}^{\hbar}$, for relatively compact subanalytic open sets $U_{j_{i}}$, we have:

$$
F^{i} \stackrel{\mathrm{w}, \hbar}{\otimes} \mathcal{C}_{X}^{\infty} \simeq \oplus_{j_{i}}\left(\mathbb{C}_{U_{j_{i}}} \stackrel{\mathrm{w}}{\otimes} \mathcal{C}_{X}^{\infty}\right)^{\hbar} \simeq \prod_{j_{i}}\left(\mathbb{C}_{U_{j_{i}}} \stackrel{\mathrm{w}}{\otimes} \mathcal{C}_{X}^{\infty}\right)^{\hbar},
$$

since the direct sum of a locally finite family of sheaves on $X_{s a}$ is isomorphic to the product of the same family. Hence, we get:

$$
\begin{gathered}
\Gamma\left(X ; F^{i} \stackrel{\mathrm{w}, \hbar}{\otimes} \mathcal{C}_{X}^{\infty}\right) \simeq \prod_{j_{i}} \Gamma\left(X ; \mathbb{C}_{U_{j_{i}}} \stackrel{\mathrm{w}}{\otimes} \mathcal{C}_{X}^{\infty}\right)^{\hbar} \simeq \\
\prod_{j_{i}}\left(\lim _{\overleftarrow{n}} \Gamma\left(X ; \mathbb{C}_{U_{j_{i}} \cap Z_{n}} \stackrel{\mathrm{w}}{\otimes} \mathcal{C}_{X}^{\infty}\right)^{\hbar}\right) \simeq \lim _{\overleftarrow{n}} \Gamma\left(X ; F_{Z_{n}}^{i} \stackrel{\mathrm{w}, \hbar}{\otimes} \mathcal{C}_{X}^{\infty}\right)
\end{gathered}
$$

As a consequence, the complex $R \Gamma\left(X ; F_{\mathrm{w}, \hbar}^{\mathrm{w}, \hbar} \mathcal{C}_{X}^{\infty}\right)$ is isomorphic to the projective limit of the complexes $\Gamma\left(X ; F_{Z_{n}}^{\bullet}{ }^{\mathrm{w}, \hbar}, \mathcal{C}_{X}^{\infty}\right)$ hence it is of FN type.

2. We shall use a similar argument as in 1.

$\left.\mathrm{a}^{\prime}\right)$ Let $U$ open subanalytic in $X$. The assertion for $F=\mathbb{C}_{U}^{\hbar}$ is an immediate consequence of Lemma 5.8.

$b^{\prime}$ ) Assume that $F$ is compactly supported and choose a resolution $F^{\bullet}$ of $F$ as in b) and apply Remark 5.11. Hence $R \Gamma_{c}\left(X ; T D b^{[\hbar]}(F)^{v}\right)$ is isomorphic to $\Gamma_{c}\left(X ; T D b^{[\hbar]}\left(F^{\bullet}\right)^{v}\right)$. Since $\Gamma_{c}(X ; \cdot)$ commutes with inductive limits, this entails that $R \Gamma_{c}\left(X ; T D b^{[\hbar]}(F)^{v}\right) \simeq W^{\bullet}$ with $W^{\bullet}$ a complex of type DFN. 
$\left.c^{\prime}\right)$ With the same choice of $Z_{n}$ as in c), note that $T D b^{[\hbar]}(F)^{v}$ is represented by the inductive limit of $T D b^{[\hbar]}\left(F_{Z_{n}}^{\bullet}\right)^{v}$. In fact, each entry $F^{i}$ of $F^{\bullet}$ is a locally finite direct sum of the form $\oplus_{j_{i}} \mathrm{C}_{U_{j_{i}}}^{\hbar}$, for subanalytic open sets $U_{j_{i}}$, and we have:

$$
\begin{gathered}
\Gamma_{c}\left(X ; T D b^{[\hbar]}\left(F^{i}\right)^{v}\right) \simeq \oplus_{j_{i}} \Gamma_{c}\left(X ; T D b^{[\hbar]}\left(\mathrm{C}_{U_{j_{i}}}\right)^{v}\right) \simeq \\
\simeq \oplus_{j_{i}}\left(\Gamma_{c}\left(X ; \operatorname{Hom}\left(\mathrm{C}_{U_{j_{i}}}, \mathcal{D} b\right)^{v}\right) \otimes \mathbb{C}_{X}^{[\hbar]}\right) \simeq \\
\simeq \oplus_{j_{i}}\left(\lim _{\vec{n}} \Gamma_{c}\left(X ; \operatorname{tHom}\left(\mathrm{C}_{U_{j_{i}} \cap Z_{n}}, \mathcal{D} b_{X}\right)^{v}\right) \otimes \mathbb{C}_{X}^{[\hbar]}\right) \simeq \lim _{\vec{n}} \Gamma_{c}\left(X ; T D b^{[\hbar]}\left(F_{Z_{n}}^{i}\right)^{v}\right),
\end{gathered}
$$

which entails that $\Gamma_{c}\left(X ; T D b^{[\hbar]}(F)^{v}\right)$ is isomorphic to a complex of type DFN.

3. Let us choose an almost free resolution of $F, F^{\bullet}$, as above, and let us choose an increasing sequence $\left\{Z_{n}\right\}_{n \in \mathbb{N}}$ of compact subanalytic subsets such that $X$ is the union of the interiors of the $Z_{n}$. Then, as we saw above, $R \Gamma\left(X ; F^{\mathrm{w}, \hbar} \stackrel{\mathcal{C}_{X}^{\infty}}{\infty}\right)\left(\operatorname{resp} . R \Gamma_{c}\left(X ; T D b^{[\hbar]}(F)^{v}\right)\right)$ is isomorphic to the complex $V^{\bullet}\left(\right.$ resp. $W^{\bullet}$ ) with entries $V^{i}=\lim _{\overleftarrow{n}} \Gamma\left(X ; F_{Z_{n}}^{i} \stackrel{\text { w,h }}{\otimes} \mathcal{C}_{X}^{\infty}\right.$ ) (resp. $\left.W^{-i}=\lim _{\vec{n}} \Gamma_{c}\left(X ; T D b^{[\hbar]}\left(F_{Z_{n}}^{i}\right)^{v}\right)\right)$ and the morphisms $v^{i}: V^{i} \rightarrow V^{i+1}$ (resp. $\left.w^{i}: W^{-i-1} \rightarrow W^{-i}\right)$ is induced by the morphisms $v_{n}^{i}: \Gamma\left(X ; F_{Z_{n}}^{i} \stackrel{\mathrm{w}, \hbar}{\otimes} \mathcal{C}_{X}^{\infty}\right) \rightarrow$ $\Gamma\left(X ; F_{Z_{n}}^{i+1} \stackrel{\mathrm{w}, \hbar}{\otimes} \mathcal{C}_{X}^{\infty}\right)\left(\right.$ resp. $\left.w_{n}^{i}: \Gamma_{c}\left(X ; T D b^{[\hbar]}\left(F_{Z_{n}}^{i+1}\right)^{v}\right) \rightarrow \Gamma_{c}\left(X ; T D b^{[\hbar]}\left(F_{Z_{n}}^{i}\right)^{v}\right)\right)$.

On the other hand, since, for each $i$ and $n$, the topological dual of $\Gamma\left(X ; F_{Z_{n}}^{i} \stackrel{\mathrm{w}, \hbar}{\otimes} \mathcal{C}_{X}^{\infty}\right)$ is $\Gamma_{c}\left(X ; T D b^{[\hbar]}\left(F_{Z_{n}}^{i}\right)^{v}\right)$, it follows that the topological dual of $V^{i}$ is $W^{-i}$ and that $w^{i}$ is the transpose of $v^{i}$. q.e.d.

Let now $X$ be a complex manifold of dimension $d_{X}$, let $\mathcal{C}_{X}^{\infty,(0, \bullet)}$ denote the complex of conjugate differential forms with coefficients in $\mathcal{C}_{X}^{\infty}$ and recall that

$$
F^{\mathrm{w}, \hbar} \mathcal{C}_{X}^{\infty,(0, \bullet)}:=\left(F^{\mathrm{w}, \hbar} \mathcal{C}_{X}^{\infty}\right) \otimes_{\mathcal{A}_{X}} \mathcal{C}_{X}^{\infty,(0, \bullet)}
$$

Then, for any $F \in D_{\mathbb{R}-c}^{b}\left(\mathbb{C}_{X}^{\hbar}\right)$,

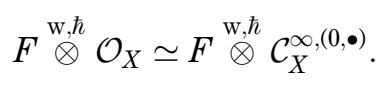

Indeed, by taking an almost free resolution of $F$, we may assume that $F=G^{\hbar}$, with $G \in \operatorname{Mod}_{\mathbb{R}-c}\left(\mathbb{C}_{X}\right)$. In that case,

$$
F \stackrel{\mathrm{w}, \hbar}{\otimes} \mathcal{O}_{X} \simeq G^{\hbar} \stackrel{\mathrm{w}, \hbar}{\otimes} \mathcal{O}_{X} \simeq\left(G \stackrel{\mathrm{w}}{\otimes} \mathcal{O}_{X}\right)^{\hbar} \simeq\left(G \stackrel{\mathrm{w}}{\otimes} \mathcal{C}_{X}^{\infty,(0, \bullet)}\right)^{\hbar} \simeq G^{\hbar} \stackrel{\mathrm{w}}{\otimes} \mathcal{C}_{X}^{\infty,(0, \bullet)} .
$$


Similarly, consider the resolution $\mathcal{D} b_{X}^{(n, n-\bullet)}$ of $\Omega_{X}\left[d_{X}\right]$. We set

$$
T D b^{[\hbar]}(F) \otimes_{\mathcal{A}_{X}} \mathcal{D} b_{X}^{(n, n-\bullet)}:=T D b^{[\hbar]}(F)^{(n, n-\bullet)} .
$$

Therefore, we get

$$
T H^{[\hbar]}(F)^{v}\left[d_{X}\right] \simeq T D b^{[\hbar]}(F)^{(n, n-\bullet)} .
$$

Theorem 6.2. Let $\mathcal{M} \in D_{\text {coh }}^{b}\left(\mathcal{D}_{X}^{\hbar}\right)$ and let $F, G \in D_{\mathbb{R}-c}^{b}\left(\mathbb{C}_{X}^{\hbar}\right)$. Then:

1. We can define $R \Gamma\left(X ; R \mathcal{H} \operatorname{Hom}_{\mathcal{D}_{X}^{\hbar}}\left(\mathcal{M} \stackrel{L}{\otimes}_{\mathbb{C}^{\hbar}} G, F \stackrel{\mathrm{w}, \hbar}{\otimes} \mathcal{O}_{X}\right)\right)$ as an object of $D^{b}(F N)$, functorially with respect to $\mathcal{M}, F$ and $G$.

2. We can define $R \Gamma_{c}\left(X ; T H^{[\hbar]}(F)^{v}\left[d_{X}\right] \stackrel{L}{\otimes_{\mathcal{D}_{X}}{ }^{h}}\left(\mathcal{M} \stackrel{L}{\otimes}_{\mathbb{C}^{h}} G\right)\right)$ as an object of $D^{b}(D F N)$ functorially with respect to $\mathcal{M}, F$ and $G$.

3. The objects described in (1) and (2) can be constructed in such a way that they are dual to each other.

Proof. We shall essentially follow the method of the proof of Theorem 6.1 of [7].

Applying Proposition 4.8 let us choose $\mathcal{L}(\mathcal{M})$ an almost free resolution of $\mathcal{M}$, and by Lemma 2.10, let us choose $\mathcal{L}(G), \mathcal{L}(F)$ almost free resolutions of $G$ and $F$ respectively. Note that the choice of these almost free resolutions are functorial as a consequence of the results of [7].

1. Note that, for any $H \in \operatorname{Mod}_{\mathbb{R}-c}\left(C_{X}\right)$ and any $k \geq 0$, $\Gamma\left(X ; \mathcal{H o m}_{\mathcal{D}_{X}^{\hbar}}\left(\mathcal{L}(\mathcal{M}) \otimes_{\mathrm{C}_{X}^{\hbar}} \mathcal{L}(G), H^{\hbar} \stackrel{\mathrm{w}, \hbar}{\otimes} \mathcal{C}_{X}^{\infty,(0, k)}\right)\right) \simeq \Pi_{i, j} \Gamma\left(U_{i} \cap V_{j} ; H^{\hbar} \stackrel{\mathrm{w}, \hbar}{\otimes} \mathcal{C}_{X}^{\infty,(0, k)}\right)$, for adequate choice of open subanalytic relatively compact open sets $U_{i}$ and $V_{j}$ (defined by $\mathcal{L}(\mathcal{M})$ and $\mathcal{L}(G)$ ).

We have

$$
\begin{aligned}
R \Gamma\left(X ; R \mathcal{H} \operatorname{Hom}_{\mathcal{D}_{X}^{h}}\left(\mathcal{M} \stackrel{L}{\otimes}_{\mathbb{C}_{X}^{\hbar}} G, F \stackrel{\mathrm{w}, \hbar}{\otimes} \mathcal{O}_{X}\right)\right) \simeq \\
\simeq \Gamma\left(X ; \mathcal{H} \operatorname{Hom}_{\mathcal{D}_{X}^{\hbar}}\left(\mathcal{L}(\mathcal{M}) \otimes_{\mathrm{C}_{X}^{h}} \mathcal{L}(G), \mathcal{L}(F) \stackrel{\mathrm{w}, \hbar}{\otimes} \mathcal{C}_{X}^{\infty,(0, \bullet)}\right)\right)
\end{aligned}
$$

The result then follows by 1 . of Proposition 6.1.

2. The proof goes similar to the proof of 1 . Replace $T H^{[\hbar]}(F)^{v}\left[d_{X}\right]$ by the isomorphic complex $T D b^{[\hbar]}(F)^{(n, n-\bullet)}$.

Noting that, for any $H \in \operatorname{Mod}_{\mathrm{R}-c}\left(\mathbb{C}_{X}\right)$, $\Gamma_{c}\left(X ; T D b^{[\hbar]}\left(H^{\hbar}\right)^{(n, n-k)} \otimes_{\mathcal{D}_{X}^{\hbar}}\left(\mathcal{L}(\mathcal{M}) \otimes_{\mathbb{C}_{X}^{\hbar}} \mathcal{L}(G)\right)\right) \simeq \oplus_{i, j} \Gamma_{c}\left(U_{i} \cap V_{j} ; T D b^{[\hbar]}\left(H^{\hbar}\right)^{(n, n-k)}\right)$ the proof follows by 2. of Proposition 6.1. 
3. The proof follows straightforwardly from 1 . and 2 . above and 3 . of Proposition 6.1.

q.e.d.

Example 6.3. Let us consider the Example 8.5 of [1]. Let $X$ be $\mathbb{R}$ with the coordinate $x$ and consider the coherent $\mathcal{D}_{X}^{\hbar}$-module defined by the equation $x-\hbar \partial_{x}, \mathcal{M}=\mathcal{D}_{X}^{\hbar} /\left\langle x-\hbar \partial_{x}\right\rangle$. As proved in loc.cit, $\mathcal{M}$ is isomorphic as a $\mathcal{D}_{X}^{\hbar}$-module to $\mathcal{N}=\mathcal{D}_{X}^{\hbar} /\langle x\rangle$.

Let $F=\mathbb{C}_{Z}$, where $Z=\{0\}$. Then $\mathbb{C}_{\{0\}}^{\hbar} \stackrel{\text { w, }, \hbar}{\otimes} \mathcal{C}_{X}^{\infty}$ is given by the exact sequence

$$
0 \rightarrow\left(\mathcal{I}_{X,\{0\}}^{\infty}\right)^{\hbar} \rightarrow \mathcal{C}_{X}^{\infty, \hbar} \rightarrow \mathbb{C}_{\{0\}}^{\hbar} \stackrel{\mathrm{w}, \hbar}{\otimes} \mathcal{C}_{X}^{\infty} \rightarrow 0
$$

Recall that a section of $\mathbb{C}_{\{0\}}^{\hbar} \stackrel{\mathrm{w}}{\otimes} \mathcal{C}_{X}^{\infty}$ is given by $\left(\lambda_{j}\right)_{j \geq 0}, \lambda_{j} \in$ C corresponding to $f \in \mathcal{C}_{X}^{\infty}$ satisfying $\partial_{x}^{(j)}(f)(0)=\lambda_{j}$. The action of $x$ on $\mathbb{C}_{\{0\}}^{\hbar} \stackrel{\mathrm{w}, \hbar}{\otimes} \mathcal{C}_{X}^{\infty} \simeq$ $\left(\mathbb{C}_{\{0\}}^{\hbar} \stackrel{\mathrm{W}}{\otimes} \mathcal{C}_{X}^{\infty}\right)^{\hbar}$ is induced by the action of $x$ in $\mathbb{C}_{\{0\}} \stackrel{\mathrm{W}}{\otimes} \mathcal{C}_{X}^{\infty}$ which is given by $x\left(\lambda_{j}\right)=\left(\mu_{j}\right)=\left(j \lambda_{j-1}\right)$ for $j \geq 0$. Therefore,

$$
\Gamma\left(X ; \mathcal{H o m}_{\mathcal{D}_{X}^{k}}\left(\mathcal{M}, \mathrm{C}_{\{0\}}^{\hbar} \stackrel{\mathrm{w}, \hbar}{\otimes} \mathcal{C}_{X}^{\infty}\right)\right)=0
$$

and

$$
\Gamma\left(X ; \mathcal{E} x t_{\mathcal{D}_{X}^{h}}^{1}\left(\mathcal{M}, \mathrm{C}_{\{0\}}^{\hbar} \stackrel{\mathrm{w}, \hbar}{\otimes} \mathcal{C}_{X}^{\infty}\right)\right) \simeq \mathbb{C}^{\hbar} .
$$

On the other hand, the action of $x$ on $\Gamma_{\{0\}}(\mathcal{D} b)^{[\hbar]}$ is induced by the action of $x$

on $\Gamma_{\{0\}}\left(\mathcal{D} b_{X}\right)$ which is given by $x \sum_{i \geq 0} \lambda_{i} \delta^{(i)}(x)=\sum_{i \geq 1} \lambda_{i}(-i) \delta^{(i-1)}(x)$.
Hence

$$
\Gamma\left(X ; \mathcal{H o m}_{\mathcal{D}_{X}^{h}}\left(\mathcal{M}, \Gamma_{\{0\}}\left(\mathcal{D} b_{X}^{[\hbar]}\right)\right)\right) \simeq(\mathrm{C} \delta(x))^{[\hbar]}
$$

and $\Gamma\left(X ; \mathcal{E} x t_{\mathcal{D}_{X}^{h}}^{1}\left(\mathcal{M}, \Gamma_{\{0\}}\left(\mathcal{D} b_{X}^{[\hbar]}\right)\right)\right)=0$.

\section{REFERENCES}

[1] A. D’Agnolo - S. Guillermou - P. Schapira, Regular holonomic $\mathcal{D}[[\hbar]]-$ modules, Publ. RIMS, Kyoto Univ., 47 (2011), pp. 221-255.

[2] A. Grothendieck, Produits tensoriels topologiques et espaces nucléaires, Mem. Amer. Math. Soc., 16, (1955).

[3] M. Kashiwara, D-modules and microlocal Calculus, Translations of Mathematical Monographs, AMS, 217, (2003).

[4] M. Kashiwara, The Riemann-Hilbert problem for holonomic systems, Publ. RIMS, Kyoto Univ., 20 (1984), pp. 319-365. 
[5] M. Kashiwara - P. SchapIRA, Ind-Sheaves, Astérisque, Soc. Math. France, 271, (2001).

[6] M. KASHIWARA - P. SchaPIRA, Sheaves on manifolds, grundlehren der Math. Wiss., 292 (Springer-Verlag, 1990).

[7] M. KASHIWARA - P. SCHAPIRA, Moderate and formal cohomology associated with constructible sheaves, Mem. Soc. Math. France, 64, (1996).

[8] M. Kashiwara - P. SchapiRa, Deformation quantization modules, Astérisque, Soc. Math. France (2012), arXiv:1003.3304.

[9] L. Prelli, Sheaves on subanalytic sites, Rend. Sem. Mat. Univ. Padova, 120 (2008).

[10] D. RAImUndo, Elliptic pairs over C[[ $\hbar]]$, submitted, arxiv:1003.4873..

[11] L. Schwartz, Espaces Nucléaires. Propriétés de permanence et exemples, Séminaire Schwartz, 1, exp. 18 (1953-1954), pp. 1-5.

Manoscritto pervenuto in redazione il 17 dicembre 2010. 